\title{
The FDG-Uptake of Adipose Tissue is Higher in Individuals with Increased Blood Glucose Levels than in Individuals with Normal Levels
}

Henry Lindholm ${ }^{1 *}$, Per Grybäck ${ }^{1}$, Alejandro Sánchez-Crespo ${ }^{2}$, Fredrik Brolin ${ }^{2}$ and Hans Jacobsson ${ }^{1}$

${ }^{1}$ Department of Radiology, Karolinska University Hospital Solna, SE-171 76 Stockholm, Sweden

${ }^{2}$ Department of Hospital Physics, Karolinska University Hospital Solna, SE-171 76 Stockholm, Sweden

"Corresponding author: Henry Lindholm, Department of Radiology, Karolinska University Hospital Solna, SE-171 76 Stockholm, Sweden, Tel: +46 70626 6960; Email: henry.lindholm@karolinska.se

Received date: Jan 02, 2014, Accepted date: March 10, 2014, Published date: March 18, 2014

Copyright: (c) 2014 Lindhlom et al. This is an open-access article distributed under the terms of the Creative Commons Attribution License, which permits unrestricted use, distribution, and reproduction in any medium, provided the original author and source are credited.

\begin{abstract}
Objective: The influence of hyperglycaemia on the FDG uptake of regular adipose tissue at PET examinations was studied in clinical patients.

Methods: The report constitutes a sub-study of 500 consecutive clinical examinations evaluated in retrospect. The inclusion criteria comprised examinations with a normal, or a near normal FDG distribution. Patients who had been subjected to chemotherapy or radiotherapy $<4$ weeks prior to the examination were excluded. Otherwise, inclusion was made irrespective of concurrent diseases and/or therapy. In the current study, a sub-group of 62 patients with increased blood glucose levels $(\geq 7.0 \mathrm{mmole} / \mathrm{l})$ was compared with another 62 patients being paired with regard to age and gender and showing blood glucose levels within normal range $(\leq 6.0 \mathrm{mmole} / \mathrm{l})$. SUVmean of the axillary fat, perirenal fat, abdominal subcutis and gluteal fat were assessed. The gluteal fat was adequate for reliable evaluations. The other regions turned out more or less consistent for this because of the partial volume phenomenon, but were included because of the large number of observations, as well as no systematic influence on the outcome could be expected.
\end{abstract}

Results: There was a weak, but significantly higher FDG-uptake of all assessed adipose tissue depots in the patients showing increased blood glucose levels compared to the patients with normal blood glucose levels. The tracer uptake also varied between the different fat depots. This is in concordance with previous studies on insulinstimulated FDG-uptake showing differences of insulin resistance between various adipose tissue depots.

Conclusion: The uptake of FDG of adipose tissue is weakly increased in individuals with hyperglycemia compared to normal individuals. The differences are so small that they can be ignored in clinical practice.

Keywords: Blood glucose level; FDG/PET; Adipose tissue; SUV; FDG distribution

\section{Introduction}

At FDG PET examination there is believed to be a competition between endogenous blood glucose and administered FDG, giving rise to a reduced uptake of pathological lesions in patients with an increased blood glucose level (B-glucose). This has led to recommendations both in the European [1] and in the American [2] guidelines to measure B-glucose prior to FDG PET examinations and reschedule patients with values exceeding a certain level. While initially adhering to the recommendations, we encountered patients with elevated B-glucose levels who for various reasons were difficult to reschedule why they were examined despite the increase. At visual evaluation, the image quality of these examinations did not differ from the quality obtained in patients with a normal B-glucose. This has increasingly caused us to accept such patients for clinical examinations.

There are a number of reports on the effect of hyperglycemia and/or diabetes mellitus on the image quality at FDG PET, some with opposing findings $[3,4]$. In addition, all such studies have focused on the tracer uptake by the pathological lesions. The uptake by surrounding normal tissues has been little studied, even though this uptake also is involved in the glucose balance. It may also comprise the background activity from which a lesion has to be discerned. In a previous comprehensive study we have in retrospect correlated SUV's of a number of normal tissues/organs with B-glucose in clinical patients showing a normal, or near normal FDG-distribution [4]. There was no correlation with the uptake by the liver, spleen, lungs, blood or the bone marrow, while a weak positive correlation with the uptake of the skeletal muscles was found. Also comparing one subgroup of the studied individuals having increased B-glucose with another subgroup matched with regard to age and gender and having normal B-glucose, showed a higher muscular uptake of FDG in the patients with increased levels.

The effect of B-glucose on the FDG-uptake of regular adipose tissue has not been investigated. In the current study, the FDG-uptake of various fat depots has been compared between the described two subgroups of individuals from our previous study. 
Citation: Lindholm H, Grybäck P, Crespo AS, Brolin F, Jacobsson H (2014) The FDG-Uptake of Adipose Tissue is Higher in Individuals with Increased Blood Glucose Levels than in Individuals with Normal Levels. J Nucl Med Radiat Ther 5: 167. doi: $10.4172 / 2155-9619.1000167$

Page 2 of 4

\section{Patients and Methods}

\section{Study design}

The current investigation constitutes a sub-study of a previously collected material [4]. In this, 500 consecutive PET/CT FDGexaminations with a normal, or a near normal activity distribution were retrospectively evaluated. Patients who had been subjected to chemotherapy (including GSF-treatment) or radiotherapy $<4$ weeks prior to the examination were excluded. Otherwise, patients were included irrespective of concurrent diseases and/or therapy. Examples of near normal activity distributions are small, probably benign lung lesions or radiation portals with an FDG-uptake not exceeding normal soft tissue activity, apparent reactive normal-sized lymph nodes with a moderately increased uptake, a weak uptake in surgical scars and accumulation in minor, presumably benign skin lesions. Patients with a minor FDG extravasation were studied, while individuals showing larger extravasations were excluded. Similarly, only patients with a minute brown adipose tissue uptake were included, although these regions were not included at the evaluation. Patients with an apparent generally increased muscular uptake as previously studied by us were not included [5]. No patient with any evidence of an active malignancy was studied.

In the current investigation, a subgroup of our previous study [4] constituting of 62 patients with a B-glucose $\geq 7.0 \mathrm{mmole} / \mathrm{l}$ (increased) paired with 62 patients with a B-glucose $\leq 6.0 \mathrm{mmole} / \mathrm{l}$ (normal) were analysed with regard the FDG uptake of various regular fat depots.

\section{Patients}

The study comprised $62+62$ patients paired with regard to age and gender. The mean age was 65 years (range 25-80), including 33 males and 29 females in each group. In the patients with an increased Bglucose, the mean B-glucose was 9.0 ( \pm SD 2.0) mmole/l and the mean body mass index (BMI) was $26.5 \mathrm{~kg} / \mathrm{m}^{2}( \pm$ SD 4.9$) .26$ of these patients were known diabetics, 13 of whom were on insulin treatment. In the controls the mean B-glucose was $5.2 \mathrm{mmole} / \mathrm{l}( \pm \mathrm{SD} 0.6)$ and the mean BMI was $24.3 \mathrm{~kg} / \mathrm{m}^{2}( \pm$ SD 3.3). One of these patients was a known diabetic on insulin treatment. This retrospective investigation was approved by the regional ethics research committee.

\section{PET/CT examinations}

B-glucose was measured immediately prior to administration of FDG using the same glucometer; HemoCueÒ Glucose 201+ (Hemocue AB, Ängelholm, Sweden).

A Biograph 64 TruePoint TrueV PET/CT scanner (Siemens Medical Solutions, Erlangen, Germany) was used. All patients fasted for at least $4 \mathrm{~h}$ prior to the examination. $4 \mathrm{MBq} / \mathrm{kg}$ body weight of [18F]-2-fluoro-2-deoxy-D-glucose (FDG) was administered i.v. and after 50-70 min rest examination was made. This usually included the middle skull to the proximal thigh. Prior to examinations with i.v. contrast medium a low-dose CT was performed for attenuation and scatter correction. Directly upon this, the PET-examination was made followed by a full-dose CT with i.v. contrast medium. At examinations without i.v. contrast medium the full-dose CT was used for attenuation and scatter correction.

The CT-examinations were performed with a tube tension of 120 $\mathrm{kV}$, a pitch of 0.8 , a slice thickness of $1.2 \mathrm{~mm}$, a rotation speed of $0.5 \mathrm{~s}$. In the diagnostic examinations the current was set to 160 ref. $\mathrm{mAs}$ and dose modulation (CARE Dose4D) was applied. In the examinations done only for photon attenuation and scattering correction the current was $50 \mathrm{mAs}$. CT-acquisitions were always done with the breathholding technique at a mean inspiratory level.

PET acquisition was performed during $3 \mathrm{~min}$ at each bed position during normal tidal breathing. The PET data were subsequently reconstructed with the manufacturer's 2D-OSEM algorithm (4 iterations and 8 subsets) using a 5 -mm post-reconstruction Gaussian filter. The image matrix size was $168 \times 168$ with a slice thickness of 5 $\mathrm{mm}$. In addition to attenuation and scatter correction, all data were corrected for dead-time and random coincidences.

\section{Image analysis}

Activity quantification was done in the PET-images using Siemens syngo Multi Modality Workplace (syngo MMWP, VE36A) by manually allocating Volumes-Of-Interest (VOI's) in the images based on the combined information from the CT- and PET-acquisitions. VOI's were bilaterally allocated of the axillary fat, perirenal fat and gluteal fat and the values in pairs averaged. The abdominal subcutaneous fat was assessed by allocating one VOI in the anterior mid-line. No active brown fat was included at any evaluation. Analysing the axillae, perirenal regions and abdominal subcutis, VOI's of very different sizes and shapes adapted to the various anatomical regions had to be defined, while the gluteal fat could be stereotypically assessed by an elliptical VOI with a volume of $5-25 \mathrm{~cm}^{3}$. SUV mean based on body weight (SUVmean-bw) as well as based on lean body mass (SUVmean-lbm) were calculated.

\section{Statistical methods}

Analysis of the data distribution showed that the Mann-Whitney $U$ test was adequate for evaluation. $\mathrm{P}<0.05$ was considered statistically significant.

\section{Results}

Comparing SUVmean-bw between the two groups, there was a significantly higher FDG uptake of all studied adipose depots in the hyperglycaemic individuals than in the normal individuals (Table 1). Comparing SUVmean-lbm, a significantly higher FDG uptake of the abdominal subcutis, prerirenal fat and gluteal fat remained in the hyperglycaemic individuals, although the differences turned out 'less' significant (Table 2). There was still higher uptake of the axillary adipose tissue in the hyperglycaemic individuals, but the difference was not significant. Another observation at both evaluations is the different uptake between the different fat depots within the individuals.

\begin{tabular}{|l|l|l|l|l|}
\hline & Axillary fat & Abdominal subcutis & Perirenal fat & Gluteal fat \\
\hline Increased B-glucose & $0.25(0.08)$ & $0.30(0.11)$ & $0.39(0.13)$ & $0.21(0.08)$ \\
\hline
\end{tabular}


Citation: Lindholm H, Grybäck P, Crespo AS, Brolin F, Jacobsson H (2014) The FDG-Uptake of Adipose Tissue is Higher in Individuals with Increased Blood Glucose Levels than in Individuals with Normal Levels. J Nucl Med Radiat Ther 5: 167. doi: $10.4172 / 2155-9619.1000167$

Page 3 of 4

\begin{tabular}{|l|l|l|l|l|}
\hline Significance & $*$ & $* * *$ & $* * *$ & $* *$ \\
\hline Normal B-glucose & $0.22(0.08)$ & $0.22(0.10)$ & $0.31(0.10)$ & $0.17(0.06)$ \\
\hline
\end{tabular}

Table 1: Mean SUV mean $( \pm \mathrm{SD})$ calculated from the body weight of various fat depots in 62 patients with increased blood glucose concentrations $(\geq 7.0 \mathrm{mmole} / \mathrm{l})$ and 62 patients with normal blood glucose concentrations $(\leq 6.0 \mathrm{mmole} / \mathrm{l})$ matched with regard to age and gender. $* * *=\mathrm{p}<0.001 ; * *=0.001 \leq \mathrm{p}<0.01 ; *=0.01 \leq \mathrm{p}<0.05 ; \mathrm{ns}=$ not significant.

\begin{tabular}{|l|l|l|l|l|}
\hline & Axillary fat & Abdominal subcutis & Perirenal fat & Gluteal fat \\
\hline Increased B-glucose & $0.18(0.07)$ & $0.19(0.10)$ & $0.26(0.11)$ & $0.14(0.09)$ \\
\hline Significance & ns & ** & $* *$ & $*$ \\
\hline Normal B-glucose & $0.16(0.06)$ & $0.15(0.08)$ & $0.22(0.08)$ & $0.12(0.05)$ \\
\hline
\end{tabular}

Table 2: Mean SUVmean $( \pm \mathrm{SD})$ calculated from the lean body mass of various fat depots in 62 patients with increased blood glucose concentrations $(\geq 7.0 \mathrm{mmole} / \mathrm{l})$ and 62 patients with normal blood glucose concentrations $(\leq 6.0 \mathrm{mmole} / \mathrm{l})$ matched with regard to age and gender. $* * *=\mathrm{p}<0.001 ; * *=0.001 \leq \mathrm{p}<0.01 ; *=0.01 \leq \mathrm{p}<0.05 ; \mathrm{ns}=$ not significant.

\section{Discussion}

Normal phenomena should ideally be studied in normal individuals, i.e. healthy volunteers. As this was not possible in the current study where a large number of observations were necessary, clinical patients showing a normal, or near normal activity distribution had to serve as subjects. All previous similar studies in humans have also been made in clinical patients. Since the FDG-distribution seems to be a zero-sum game [5], only patients with a normal PET-finding should ideally be studied. This is difficult since few clinical patients show a completely normal FDG-distribution. Consequently, examinations showing a minor diverging uptake of an extent assumed negligibly affecting the activity distribution also had to be included.

SUV-assessments are strongly influenced by the partial volume phenomenon, which in small lesions will lead to an underestimation of the uptake. In the current PET-system, a lesion corresponding to a sphere with diameter of $\geq 2 \mathrm{~cm}\left(4.2 \mathrm{~cm}^{3}\right)$ is necessary for a complete recovery of the activity. Of the different adipose regions assessed, only the gluteal fat was sufficiently large to consequently allow a completely reliable evaluation. The other studied regions were not large enough in all patients to escape the partial volume phenomenon. Due to the same mechanism, there may in some of these fat depots also be an activity spill-over from adjacent lean tissues with a more pronounced FDGuptake giving rise to a higher calculated uptake than by the adipose tissue itself. Nevertheless, the fat depots outside the gluteal fat were included in the evaluation as the large number of observations together with the comparative nature of the study much should compensate for the shortcomings. In addition, we cannot see these would in a systematic way influence our findings.

While calculation of SUV's usually is based on the body weight, it may be more adequate to base this on the lean body mass as little FDG is accumulated in adipose tissue. Consequently, SUVmean-lbm may constitute a 'sharper' assessment. At this, the statistical power between the two studied groups turned out 'weaker' but the outcome was, in principle, not different.

Previous studies have convincingly shown a reduction of the FDG uptake of the brain at increased B-glucose [6-8]. The opposite effect on the myocardial uptake of FDG is well known [9]. Outside these tissues, previous studies of the effects of B-glucose and/or diabetes on the distribution of FDG in normal tissues are not consistent. Summarizing the literature, in case of an effect by increased B-glucose a higher uptake by normal tissues outside the brain is always reported. It also seems that the FDG uptake of normal tissues is more affected by acute (experimentally induced) hyperglycemia than by chronic hyperglycemia [4]. Adipose tissue has not been included at these basic nuclear medicine studies. Probably this has been considered to be of limited interest as FDG very little distributes into fat [10]. This is, however, deceptive. Even if fat does not directly accumulate FDG, the adipocytes themselves, representing a small fraction of the assessed tissue, are highly responsive to insulin [11]. Consequently, much lower SUV's than usually had to be dealt with in the current study, which actually showed uptake levels of such an extent that differences between the groups could be detected and allow conclusions.

Examinations with FDG-PET offer a feasible tool to investigate the glucose metabolism [12]. Basic studies on insulin-stimulated FDGuptake have shown differences of insulin resistance between various adipose tissue depots in health and in disease. Excess amounts of abdominal subcutaneous and of visceral fat are associated with insulin resistance, while gluteal-femoral fat has been hypothesized to offer protection against insulin resistance [11-14]. Despite the possibility to precisely compare the tracer uptake of the different regions of the current study is limited as described, our findings strongly indicate a certain functional difference between the various adipose regions. Especially, there has to be a higher uptake of the perirenal fat compared to the other regions. This may reflect the same mechanism as the reported differences of insulin resistance between different adipose depots [11-14]. Nevertheless, the direct effect on the FDGuptake by the B-glucose level of adipose tissue has not been previously studied, why our findings contribute to the knowledge of the factors governing the distribution of FDG.

The higher FDG uptake of adipose tissue with increasing B-glucose is not consistent with the concept of a straight competition between FDG and endogenous glucose giving rise to a lower tracer uptake of various 'indifferent' tissues. The cellular glucose uptake is a complex mechanism influenced by several insulin-dependent as well as noninsulin-dependent factors, and a thorough analysis of these is beyond the scope of this work. Like glucose, FDG is transferred into the cells mediated by the glucose transporters 1-7 (Glut1-Glut7) and the 
Citation: Lindholm H, Grybäck P, Crespo AS, Brolin F, Jacobsson H (2014) The FDG-Uptake of Adipose Tissue is Higher in Individuals with Increased Blood Glucose Levels than in Individuals with Normal Levels. J Nucl Med Radiat Ther 5: 167. doi: $10.4172 / 2155-9619.1000167$

Page 4 of 4

sodium-glucose-linked transporters 1-2 [3]. Most glucose transporters are expressed in a tissue-specific manner. Interestingly, there is a large expression of Glut-4 transporters in fat, skeletal muscle and heart $[15,16]$, these tissues also showing an increased FDG-activity at increased B-glucose.

\section{Conclusion}

The uptake of FDG in adipose tissue is increased in individuals with hyperglycemia compared to normal individuals. This adds to our knowledge of the factors controlling the glucose distribution in health and disease. The effect is weak and together with all factors affecting the image quality at FDG/PET-examination in the individual patient, it can be disregarded in clinical practice.

\section{Acknowledgment}

The authors wish to thank Elisabeth Berg, B.Sc., Karolinska Institutet, for the professional statistical analyses.

\section{References}

1. Boellaard R, O'Doherty MJ, Weber WA, Mottaghy FM, Lonsdale MN, et al. (2010) FDG PET and PET/CT: EANM procedure guidelines for tumour PET imaging: version 1.0. Eur J Nucl Med Mol Imaging 37: 181-200.

2. Delbeke D, Coleman RE, Guiberteau MJ, Brown ML, Royal HD, et al. (2006) Procedure guideline for tumor imaging with 18F-FDG PET/CT 1.0. J Nucl Med 47: 885-895.

3. Rabkin Z, Israel O, Keidar Z (2010) Do hyperglycemia and diabetes affect the incidence of false-negative 18F-FDG PET/CT studies in patients evaluated for infection or inflammation and cancer? A Comparative analysis. J Nucl Med 51: 1015-1020.

4. Lindholm H, Brolin F, Jonsson C, Jacobsson H (2013) The relation between the blood glucose level and the FDG uptake of tissues at normal PET examinations. EJNMMI Res 3: 50.

5. Lindholm H, Johansson O, Jonsson C, Jacobsson H (2012) The distribution of FDG at PET examinations constitutes a relative mechanism: significant effects at activity quantification in patients with a high muscular uptake. Eur J Nucl Med Mol Imaging 39: 1685-1690.

6. Yamada K, Endo S, Fukuda H, Abe Y, Yoshioka S, et al. (1985) Experimental studies on myocardial glucose metabolism of rats with 18F-2-fluoro-2-deoxy-D-glucose. Eur J Nucl Med 10: 341-345.

7. Wahl RL, Henry CA, Ethier SP (1992) Serum glucose: effects on tumor and normal tissue accumulation of 2-[F-18]-fluoro-2-deoxy-D-glucose in rodents with mammary carcinoma. Radiology 183: 643-647.

8. Hara T, Higashi T, Nakamoto Y, Suga T, Saga T, et al. (2009) Significance of chronic marked hyperglycemia on FDG-PET: is it really problematic for clinical oncologic imaging? Ann Nucl Med 23: 657-669.

9. Beanlands RSB, Thorn S, Dasilva J, Ruddy TD, Maddahi J (2009) Myocardial viability. In Principle and practice of PET and PET/CT. (2ndedn), Edited by Wahl RL. Philadelphia: Lippincott Williams \& Wilkins 565-588.

10. Adler LP, Blair HF, Williams RP, Pathria MN, Makley JT, et al. (1990) Grading liposarcomas with PET using [18F]FDG. J Comput Assist Tomogr 14: 960-962.

11. Ng JM, Azuma K, Kelley C, Pencek R, Radikova Z, et al. (2012) PET imaging reveals distinctive roles for different regional adipose tissue depots in systemic glucose metabolism in nonobese humans. Am J Physiol Endocrinol Metab 303: E1134-1141.

12. Virtanen KA, Peltoniemi P, Marjamäki P, Asola M, Strindberg L, et al. (2001) Human adipose tissue glucose uptake determined using [(18)F]fluoro-deoxy-glucose ([(18)F]FDG) and PET in combination with microdialysis. Diabetologia 44: 2171-2179.

13. Virtanen KA, Lönnroth P, Parkkola R, Peltoniemi P, Asola M, et al. (2002) Glucose uptake and perfusion in subcutaneous and visceral adipose tissue during insulin stimulation in nonobese and obese humans. J Clin Endocrinol Metab 87: 3902-3910.

14. Torriani M, Zanni MV, Fitch K, Stavrou E, Bredella MA, et al. (2013) Increased FDG uptake in association with reduced extremity fat in HIV patients. Antivir Ther 18: 243-248.

15. Gould GW, Holman GD (1993) The glucose transporter family: structure, function and tissue-specific expression. Biochem J 295 : 329-341.

16. Ismail-Beigi F (1993) Metabolic regulation of glucose transport. J Membr Biol 135: 1-10. 